\title{
Microvascular inflammatory responses to ceramide and cigarette smoke in the intact rat assessed with intravital two-photon microscopy
}

\author{
MB Brown, AJ Fisher, RM Sandoval, MJ Justice, BA Molitoris, RG Presson, I Petrache \\ School of Health and Rehabilitation Sciences, School of Medicine \\ Indiana University - Purdue University Indianapolis
}

\begin{abstract}
We hypothesized that ceramide is a mediator of early alveolar lung injury induced by cigarette smoke, characterized by alterations of the alveolar barrier function. We investigated this hypothesis by utilizing a novel application of intravital two-photon excitation microscopy (TPM) of the lung in a living, breathing animal.

Methods: We first developed a technique of TPM to permit imaging of the lung maintained within the thoracic cavity of an intact rat. To accomplish this, we optimized the lung-microscope interface with an imaging window uniquely designed to minimize cardiac and respiratory motion during TPM acquisitions. To investigate alveolar barrier disruption in real time, we utilized intravenous (i.v.) fluorescent probes to examine changes in alveolar leukocyte trafficking and microvascular barrier function in response to i.v. ceramide (C16:0 PEG, $10 \mathrm{mg} / \mathrm{kg}$ ), and to cigarette smoke extract (CSE) delivered i.v. (1ml/kg) or intratracheally via a nebulizer $(2 \mathrm{~mL} / \mathrm{kg})$. Results: We performed intravital TPM monitoring of the lung microcirculation of a living rat with maintained physiological cardio-pulmonary parameters for up to 3h. Time-lapse and 3-D reconstruction images revealed heterogeneous extravasation of FITC-labeled serum albumin from the alveolar microcirculation into the alveolar airspaces in response to ceramide, in a dosedependent manner. Further, we noted that in response to both ceramide and to CSE, leukocytes accumulated in the lung parenchyma and demonstrated reduced mobility through the microcirculation, suggesting increased adhesion to the endothelium. Intratracheal administration of CSE caused increased extravasation of leukocytes into alveolar spaces within 10 minutes. Conclusions: We developed approaches that permit the application of intravital TPM to lung with no motion artifacts from the breathing and cardiac cycles. This approach permits visualization of the lung subpleural parenchyma with a high resolution. Both gross and subtle inflammatory changes that reflect alveolar epithelial and/or endothelial barrier dysfunction can be assessed with this methodology. Funding: R21 DA029249, T32 HL091816
\end{abstract}

